\title{
Activation of NLRP3 inflammasomes contributes to hyperhomocysteinemia-aggravated inflammation and atherosclerosis in apoE-deficient mice
}

\author{
Renqing Wang ${ }^{1,4}$, Yiqin Wang ${ }^{1,4}$, Nana Mu¹, Xiaoying Lou', Weixuan $\mathrm{Li}^{1}$, Yanming Chen², Dong Fan ${ }^{1}$ and \\ Hongmei $\operatorname{Tan}^{1,3}$
}

Hyperhomocysteinemia (HHcy) has been shown to promote vascular inflammation and atherosclerosis, but the underlying mechanisms remain largely unknown. The NLRP3 inflammasome has been identified as the cellular machinery responsible for activation of inflammatory processes. In this study, we hypothesized that the activation of NLRP3 inflammasomes contributes to $\mathrm{HHcy}$-induced inflammation and atherosclerosis. $\mathrm{ApoE}^{-/-}$mice were fed regular chow, high-fat (HF) diet, or $\mathrm{HF}$ plus high methionine diet to induce HHcy. To assess the role of NLRP3 inflammasomes in HHcy-aggravated atherosclerosis, NLRP3 shRNA viral suspension was injected via tail vein to knock down the NLRP3 gene. Increased plasma levels of IL-1 $\beta$ and IL-18, aggravated macrophage infiltration into atherosclerotic lesions, and accelerated development of atherosclerosis were detected in HHcy mice as compared with control mice, and were associated with the activation of NLRP3 inflammasomes. Silencing the NLRP3 gene significantly suppressed NLRP3 inflammasome activation, reduced plasma levels of proinflammatory cytokines, attenuated macrophage infiltration and improved HHcy-induced atherosclerosis. We also examined the effect of homocysteine (Hcy) on NLRP3 inflammasome activation in THP-1differentiated macrophages in the presence or absence of NLRP3 siRNA or the caspase-1 inhibitor Z-WEHD-FMK. We found that Hcy activated NLRP3 inflammasomes and promoted subsequent production of IL-1 $\beta$ and IL-18 in macrophages, which were blocked by NLRP3 gene silencing or Z-WEHD-FMK. As reactive oxygen species (ROS) may have a central role in NLRP3 inflammasome activation, we next investigated whether antioxidant $N$-acetyl-L-cysteine (NAC) prevented Hcy-induced NLRP3 inflammasome activation in macrophages. We found Hcy-induced NLRP3 inflammasome activation was abolished by NAC. Treatment with NAC in HHcy mice also suppressed NLRP3 inflammasome activation and improved $\mathrm{HHcy}$-induced atherosclerosis. These data suggest that the activation of NLRP3 inflammasomes contributes to $\mathrm{HHcy}$-aggravated inflammation and atherosclerosis in apoE ${ }^{-/-}$mice. Hcy activates NLRP3 inflammasomes in ROS-dependent pathway in macrophages. These results may have implication for the treatment of HHcy-associated cardiovascular diseases.

Laboratory Investigation (2017) 97, 922-934; doi:10.1038/labinvest.2017.30; published online 10 April 2017

A number of studies have demonstrated that chronic inflammation is a major driving force in atherogenesis and contributes to lesion rupture. ${ }^{1}$ Hyperhomocysteinemia (HHcy), an independent risk factor for cardiovascular disease, has been demonstrated to promote vascular inflammation and atherosclerosis. It has been shown that homocysteine (Hcy) promotes expression and secretion of monocyte chemoattractant protein-1 and IL-8 in human monocytes. ${ }^{2}$ Recent studies have shown that HHcy causes inflammatory monocyte differentiation and macrophage maturation in vessel walls, enhances vascular inflammation and accelerates atherosclerosis. $^{3}$ However, the mechanisms underlying HHcy-induced inflammation remain largely unknown.
Tschopp and colleagues first identified the inflammasome as a proinflammatory caspase activation complex in 2002. ${ }^{4}$ The inflammasome was believed as the cellular machinery responsible for activation of inflammatory processes. ${ }^{5}$ It is a molecular complex triggering activation of inflammatory caspases and processing of pro-IL- $1 \beta .{ }^{5}$ Among different types of inflammasomes, the Nod-like receptor protein 3 (NLRP3) inflammasome is well characterized in a variety of mammalian cells. The NLRP3 inflammasome consists of the NLRP3 scaffold, the adaptor protein apoptosis-associated speck-like protein containing a caspase recruitment domain (ASC) and caspase- $1 .{ }^{6}$ Upon NLRP3 activation, pro-caspase-1 clustering permits autocleavage and formation of the active caspase- 1

\footnotetext{
'Department of Pathophysiology, Zhongshan School of Medicine, Sun Yat-sen University, Guangzhou, China; 'Division of Endocrinology, Third Affiliated Hospital, Sun Yat-sen University, Guangzhou, China and ${ }^{3}$ Guangdong Engineering and Technology Research Center for Disease-Model Animals, Sun Yat-sen University, Guangzhou, China Correspondence: Professor H Tan, MD, PhD or Dr D Fan, MD, PhD, Department of Pathophysiology, Zhongshan School of Medicine, Sun Yat-sen University, Guangzhou 510080, China.

E-mail: tanhm@mail.sysu.edu.cn or fand@mail.sysu.edu.cn

${ }^{4}$ These authors contributed equally to this work.
}

Received 8 July 2016; revised 5 February 2017; accepted 6 February 2017 
p10/p20 tetramer, then causes the maturation of proinflammatory cytokines such as IL-1 $\beta$ and IL-18. ${ }^{7}$ NLRP3 inflammasomes have essential and prominent role in inflammation and innate immunity, and are closely associated with a number of human diseases, including type II diabetes, ${ }^{8}$ Alzheimer's disease ${ }^{9}$ and silicosis. ${ }^{10,11}$

NLRP3 inflammasomes were first shown to be activated by cholesterol crystals and are required for atherogenesis. ${ }^{12}$ Recent studies have shown that NLRP3 inflammasomes can also be activated by oxidized low-density lipoprotein or high glucose. ${ }^{13,14}$ Silencing of NLRP3 suppresses atherosclerosis and stabilizes plaques in vasculature of apolipoprotein E-deficient $\left(\right.$ apoE $\left.^{-/-}\right)$mice. ${ }^{15}$ All of these findings indicate that NLRP3 inflammasomes are profoundly involved in the development of atherosclerosis. However, the role of NLRP3 inflammasomes in HHcy-induced atherosclerosis remains unknown.

Hcy contains a free sulfhydryl group, which provides its redox properties. Hcy can be autooxidized with another Hcy molecule to generate a disulfide and reactive oxygen species (ROS). Oxidation has been proposed as a primary biochemical mechanism responsible for Hcy pathogenesis. Given that the generation of ROS is a common requirement for most NLRP3 activators, ${ }^{16}$ NLRP3 inflammasomes may be an attractive candidate as an initiating molecular switch to turn on the inflammatory response induced by HHcy. In this study, we investigated whether the activation of NLRP3 inflammasomes contributes to HHcy-induced inflammation and atherogenesis in $\operatorname{apoE}^{-/-}$mice. We also examined whether Hcy activates NLRP3 inflammasomes in a ROS-dependent pathway in macrophages.

\section{MATERIALS AND METHODS Animal Protocols}

Male $\mathrm{apoE}^{-/-}$mice $(20-25 \mathrm{~g}, 8$ weeks) on the C57/BL6 background were provided by Vital River Laboratory Animal Technology Co. Ltd (Beijing, China). Mice were randomized into control, high fat (HF), HHcy, HHcy+scrambled shRNA and HHcy+NLRP3 shRNA groups ( $n=8$ for each group). The control or HF mice were fed a regular diet or HF diet (TD08028, Harlan Teklad, USA) for 10 weeks, respectively. Mice in HHcy, HHcy+scrambled shRNA and HHcy+NLRP3 shRNA groups were fed a HF plus high methionine diet (HF +HM diet, TD08029, Harlan Teklad) for 10 weeks. The HF $+\mathrm{HM}$ diet is the HF diet with up to $19.56 \mathrm{~g} / \mathrm{kg}(2 \%)$ methionine. ${ }^{3}$ All mice were kept in certified specific pathogen-free facilities maintained around $24{ }^{\circ} \mathrm{C}$ with a 12-h light/dark cycle and free access to food and water. The animal experiments were approved by the Animal Care and Use Committee of Sun Yat-sen University. All animal care and procedures conform to the National Institutes of Health guidelines. At the end of each experiment, mice were anesthetized by an intraperitoneal (i.p.) injection of pentobarbital $(80 \mathrm{mg} / \mathrm{kg}$ body weight), which was increased (up to $100 \mathrm{mg} / \mathrm{kg}$ ) if the mice moved or showed pain upon monitoring. The adequacy of anesthesia was evaluated by monitoring toe-pinch reflex and regular respiration. Mice were killed and blood was collected. Plasma was separated and stored at $-80^{\circ} \mathrm{C}$ until further analysis.

\section{Lentiviral Silencing of NLRP3}

Based on the structure of the U6-MCS-Ubi-EGFP viral vector (Genechem Gene, Shanghai, China), double-stranded DNA were synthesized and inserted into a linearized vector. NLRP3-specific target sequence (5'-CCACAATTCTGAC CCACAA- $\left.3^{\prime}\right)$ and the scrambled sequence $\left(5^{\prime}\right.$-TTCTCC GAACGTGTCACGT-3') were packaged. In preliminary experiments, we examined the expression of NLRP3 by western blotting at $0,1,2,3,4$ and 5 weeks after injection of NLRP3 shRNA $\left(3 \times 10^{7}\right.$ units $)$ in C57 mice. We found a marked downregulation of NLRP3 at 1-4 weeks postinjection, which recovered after 5 weeks. To ensure the downregulation of NLRP3 was induced, two injections at week 2 and week 6 were administered in the subsequent studies. The mice that had been fed the HF+HM diet were randomly divided into $\mathrm{HHcy}(n=8), \mathrm{HHcy}+$ scrambled shRNA (negative control, $n=8$ ) and HHcy+NLRP3 shRNA (NLRP3 silencing, $n=8$ ) groups. The NLRP3 silencing mice and negative control mice were injected with $3 \times 10^{7}$ units of NLRP3 shRNA and scrambled shRNA viral suspension via tail vein twice at the second and sixth week of HF+HM diet respectively. The efficacy of dose and interval for delivering NLRP3 shRNA was optimized based on our preliminary experiments and was confirmed in this study.

\section{NAC Treatment}

To assess the role of ROS in HHcy-induced activation of NLRP3 inflammasomes in vivo, mice fed HF+HM diet were given a daily i.p. injection of either saline $(n=6)$ or antioxidant $\mathrm{N}$-acetyl-L-cysteine (NAC) (Sigma, $20 \mathrm{mg} / \mathrm{kg} /$ day, $n=8$ ) three times per week on alternate days over 10 weeks of HF+HM diet. The dose of NAC used in the study was demonstrated to reduce the severity of atherosclerosis previously. ${ }^{17}$

\section{Plasma Hcy and Lipids Measurement}

Plasma Hcy concentrations were measured by fluorescence immunoassay (Abbott IMx analyzer, Abbott Laboratories, Berkshire, UK) as previously reported. ${ }^{18}$ Plasma total cholesterol (TC) and triglyceride (TG) levels were assayed with enzymatic kits (Zhongsheng Biotechnology, Beijing, China) according to the manufacturer's instructions.

\section{Plasma IL-1 $\beta$ and IL-18 Assay}

Plasma levels of proinflammatory cytokines IL- $1 \beta$ and IL-18 were measured by multiplexed immunoassay with a commercially available kit (eBioscience, MA, USA). The procedure was performed according to the manufacturer's instructions using the Bio-Plex suspension array system (Bio-Plex 200, Bio-Rad, USA). 


\section{En Face Analysis of Aortic Lesion}

At the end of the experiment, mouse aortas were removed $2 \mathrm{~mm}$ from the heart and excised from the aortic arch to just beyond the renal artery, opened longitudinally and stained with Oil red O. The area of the atherosclerotic lesion (Oil red $\mathrm{O}$ area) was observed under a microscope (OLYMPUS BX51, Shinjuku-ku, Tokyo, Japan). Total area, atherosclerotic lesion area of the whole aorta were measured using the Image Plus program (Media Cybernetics, Silver Spring, MD, USA).

\section{Histologic Analysis of Aortic Root Plaque}

Hearts were harvested together with a short segment of aorta, stored in OCT compound and quick-frozen on dry ice. Cryostat sections $(4 \mu \mathrm{m})$ were harvested from where the three aortic valves first appeared up to where the aortic valves disappeared. Sections were stained with Oil red $\mathrm{O}$ and counterstained with hematoxylin for lesion evaluation. The percent lesion area of the aortic sinus was calculated, using the updated Image-Pro Plus program (Media Cybernetics). The average value (mean of three sections per mouse) was measured by a single observer blinded to the experimental protocol and used for analysis.

\section{Analysis of NLRP3 Inflammasome Activation in Atherosclerotic Lesion}

Aortic root sections were double immunofluorescent stained for two components of NLRP3 inflammasome. Briefly, sections were incubated with goat anti-NLRP3 (Abcam, Cambridge, UK) and rabbit anti-ASC (Adipogen, Switzerland) antibodies, or goat anti-NLRP3 and rabbit anti-caspase1 antibodies (Abcam) overnight at $4^{\circ} \mathrm{C}$. The anti-caspase- 1 antibody reacts with the $45 \mathrm{kDa}$ protein caspase- 1 (procaspase-1). After washing with phosphate-buffered saline (PBS) buffer, the slides were incubated with Alexa-647labeled and Alexa-488-labeled secondary antibodies (Abcam) for $1 \mathrm{~h}$ at room temperature. The slides were subjected to examinations using a confocal laser scanning microscope (LSM780, Zeiss, Germany) after being mounted with DAPIcontaining mounting solution.

\section{Analysis of Macrophage Accumulation and NLRP3 Inflammasome Activation in Atherosclerotic Lesion}

To assess macrophages infiltration into atherosclerotic plaques, frozen sections of aortic root were fixed, permeabilized and incubated with goat anti-NLRP3 (Abcam) and rat anti-MOMA-2 (monocyte and macrophage marker) antibodies (AbD Serotec, Raleigh, NC, USA) overnight at $4{ }^{\circ} \mathrm{C}$. After washing with PBS buffer, slides were incubated with Alexa-647-labeled and Alexa-488-labeled secondary antibodies (Abcam). The slides were subjected to examinations using a confocal laser scanning microscope (LSM780, Zeiss) after being mounted with DAPI-containing mounting solution.

\section{Cell Culture}

THP-1 cells were cultured in RPMI-1640 medium (Hyclone, USA) supplemented with $10 \%$ fetal bovine serum (FBS, Hyclone), as well as $100 \mathrm{U} / \mathrm{ml}$ penicillin and streptomycin. THP-1 cells were treated with phorbo 12 -myristatel 13-acetate $(80 \mathrm{nmol} / \mathrm{l})$ to induce the differentiation of monocytes into macrophages. Cells were subsequently washed and incubated with different concentration of Hcy (Sigma, USA) for $24 \mathrm{~h}$. In some experiments, macrophages were treated with Hcy after transfected with NLRP3 siRNA or scrambled siRNA for $4 \mathrm{~h}$; or macrophages were treated with Hcy in the presence or absence of caspase-1 inhibitor Z-WEHD-FMK ( $20 \mu \mathrm{mol} / \mathrm{l}, \mathrm{RD}$, USA), or antioxidant NAC (50 mmol/l, Sigma).

\section{NLRP3 SiRNA Transfection}

The NLRP3-specific siRNA were designed with the siRNA Selection Program (AmbionInc, TX, USA). The sequences are as follows: NLRP3 siRNA: 5'-GUGCAUUGAAGACAGGAA UTT-3' and 3'-AUUCCUGUCUUCAAUGCACTT-5', scrambled siRNA: $5^{\prime}$-UUCUCCGAACGUGUCACGUTT- $3^{\prime}$ and 3'-ACGUGACACGUUCGGAGAATT-5' (Genechem Gene). THP-1-differentiated macrophages were transfected with NLRP3 siRNA or scrambled siRNA. The NLRP3 siRNA or scrambled siRNA in serum-free opti-MEM was mixed with Lipofectamine 2000 respectively (Invitrogen, Rockville, MD, USA), according to the manufacturer's protocol. After 4-h transfection, cells were subsequently washed and incubated with Hcy $200 \mu \mathrm{mol} / \mathrm{l}$ for $24 \mathrm{~h}$. Cells were harvested for western blot or immunofluorescent staining, and supernatants were collected for ELISA.

\section{IL-1 $\beta$ and IL-18 ELISA}

IL- $\beta$ and IL-18 in cultured supernatants were measured with human IL- $1 \beta$ and IL-18 ELISA kit (eBioscience, San Diego, USA) according to the manufacturer's instructions.

\section{Analysis of NLRP3 Inflammasome Activation in Macrophages}

For colocalization of inflammasome molecules, macrophages were plated on coverslips and stimulated as described above. The cells were washed and fixed with 4\% PFA in PBS for 15 min, blocked with 10\% FBS in PBS and permeabilized with Triton X-100. The cells were double immunofluorescent stained with anti-NLRP3 (Abcam) and anti-ASC (Abcam) or anti-caspase-1 (Abcam) antibodies overnight at $4^{\circ} \mathrm{C}$. After washing with PBS, the cells were incubated with Alexa-647labeled or Alexa-488-labeled secondary antibodies (Abcam) for $1 \mathrm{~h}$ at room temperature. After being mounted with DAPI-containing mounting solution, the slides were subjected to examinations using a confocal laser scanning microscope (LSM780, Zeiss).

\section{Detection of Intracellular ROS}

Intracellular ROS levels were measured using a cell-permeable fluorescent probe, 2'7'-dichlorofluorescein diacetate 
(DCFH-DA) as instructed by the manufacturer. Macrophages were incubated with $50 \mathrm{mmol} / \mathrm{l} \mathrm{NAC}$ for $1 \mathrm{~h}$, then treated with $200 \mu \mathrm{mol} / \mathrm{l} \mathrm{Hcy}$ for $0.5 \mathrm{~h}$. Cells were incubated with fresh DCFH-DA $(10 \mu \mathrm{mol} / \mathrm{l})$ in PBS in dark for $30 \mathrm{~min}$ at $37^{\circ} \mathrm{C}$. The fluorescence was monitored with a confocal laser scanning microscope (LSM780, Zeiss).

\section{Western Blot Analysis}

Protein from macrophage lysate or mouse aortas (removed $2 \mathrm{~mm}$ from the heart and excised from the aortic arch to just beyond the renal artery) was collected. Western blot procedures were carried out as described previously ${ }^{18,19}$ with specific antibodies against NLRP3 (Adipogen), caspase-1 (Santa Cruz, USA) and IL-1 $\beta$ (Cell Signaling Technology, MA, USA). The blot was developed with an enhanced chemiluminescence reagent kit (Applygen Technologies, Beijing, China). Quantification of band intensity was carried out using Image J software (NIH, Bethesda, MD, USA).

\section{Statistical Analysis}

Results were expressed as the mean \pm s.d. All in vitro data were from at least three independent experiments. Statistical comparison among multiple groups was carried out by one-way ANOVA followed by LSD test using SPSS 17.0 software. Statistical comparison between two groups was performed via Student's $t$-test. $P<0.05$ was considered statistically significant.

\section{RESULTS}

\section{HM Diet-Induced HHcy in ApoE ${ }^{-1-}$ Mice}

The $\mathrm{HF}+\mathrm{HM}$ diet-induced HHcy in apoE ${ }^{-/-}$mice. Plasma total Hcy levels in $\mathrm{HF}+\mathrm{HM}$ diet mice were significantly increased compared with mice fed regular diet or mice fed HF diet alone (Figure 1a). Plasma levels of TC and TG were significantly increased in mice fed HF diet and HF+HM diet compared with mice fed regular diet. There was no significant difference in plasma levels of TC and TG between mice fed $\mathrm{HF}$ diet and mice with HHcy induced by $\mathrm{HF}+\mathrm{HM}$ diet (Figures $1 \mathrm{~b}$ and $\mathrm{c}$ ). Lentiviral silencing of NLRP3 had no effect on plasma levels of Hcy, TC and TG (Figures 1a-c).

\section{Lentiviral Silencing of NLRP3 Attenuated HHcy- Accelerated Atherosclerosis}

The en face Oil red $\mathrm{O}$ staining of aortas was performed to determine the lesion area covering the aortic surface. No obvious lesions were seen in the aortic tree of mice with the regular diet, whereas HF diet induced marked atherosclerosis in $\mathrm{apoE}^{-/-}$mice. Atherosclerotic lesions were mainly observed at the branch points of the aortic arch and at the ostia of the intercostal arteries in mice fed HF diet alone (Figure 2a lower panel). HHcy mice had larger atherosclerotic lesion areas in the aortic arch, thoracic and abdominal aorta when compared with mice fed the HF diet only $(P<0.05$, Figure 2a lower panel and Figure 2c). Moreover, frozen sections of the aortic root stained with Oil Red $\mathrm{O}$ also showed
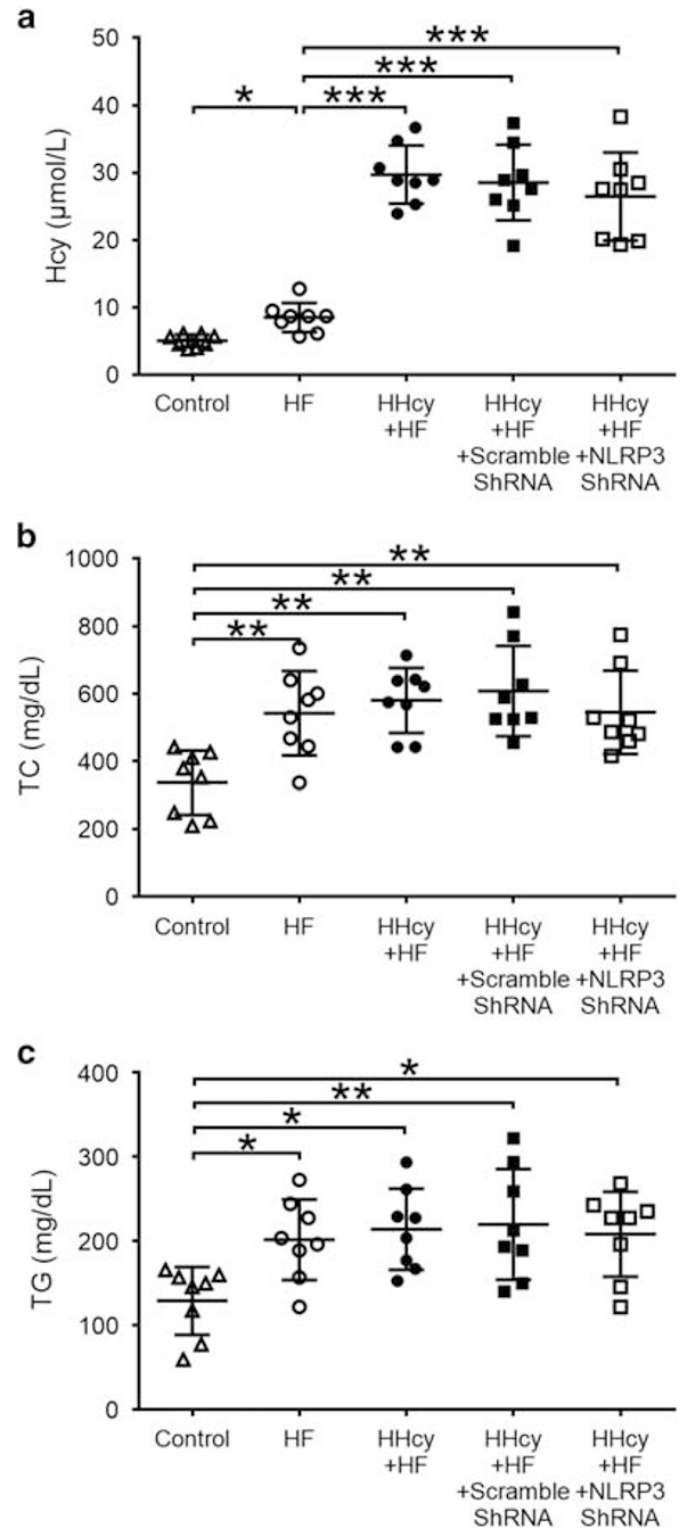

Figure 1 Plasma levels of $\mathrm{Hcy}, \mathrm{TC}$ and $\mathrm{TG}_{\mathrm{G}}$ apoE $\mathrm{E}^{-/-}$mice. ApoE $\mathrm{E}^{-/-}$mice were fed regular chow, $\mathrm{HF}$ diet or $\mathrm{HF}$ plus high methionine $(\mathrm{HF}+\mathrm{HM})$ diet to induce HHcy for 10 weeks. The NLRP3 shRNA and scrambled shRNA were injected via tail vein twice at the second and the sixth week after HF+HM diet. Plasma levels of Hcy (a), TC (b) and TG (c). Hcy, homocysteine; HHcy, hyperhomocysteinemia; TC, total cholesterol; TG, triglyceride. $n=8$. ${ }^{*} P<0.05,{ }^{*} P<0.01,{ }^{*}{ }^{*} P<0.001$.

that the atherosclerotic lesion areas in HHcy mice was significantly increased compared with that in HF diet mice (Figure 2a upper panel and Figure 2b).

To assess the role of NLRP3 inflammasomes in HHcyaggravated atherosclerosis, HHcy mice were injected with NLRP3 shRNA viral suspension to knock down NLRP3 gene. Blocking efficiency was detected by protein expression of NLRP3 (Figures 3a and b). Interestingly, silence of NLRP3 attenuated HHcy-aggravated atherosclerosis in both aortic tree and aortic root. In HHcy mice/HHcy+scrambled shRNA 
mice, approximately $6.89 \% / 7.02 \%$ of the aortic surface was covered by atherosclerotic plaques, and there was no difference between these two groups. The positive staining percentage was reduced to $4.96 \%$ by knock down NLRP3 gene in HHcy+NLRP3 shRNA mice $(P<0.05$, Figure 2a lower panel and Figure 2c). Similarly, the atherosclerotic lesion area accounted for $32.32 \% / 34.47 \%$ of the aortic sinus in HHcy mice/HHcy+scrambled shRNA mice in comparison with $25.71 \%$ in HHcy+NLRP3 shRNA mice $(P<0.05$, Figure $2 \mathrm{a}$ upper panel and Figure 2b). These findings suggest the involvement of NLRP3 inflammasomes in mediating HHcy-induced atherosclerosis.

\section{Lentiviral Silencing of NLRP3 Inhibited HHcy-Induced Activation of NLRP3 Inflammasomes}

The colocalization of NLRP3 inflammasome components (NLRP3, ASC and caspase-1) has been considered as a marker for assessing inflammasome activation. ${ }^{20}$ As shown in
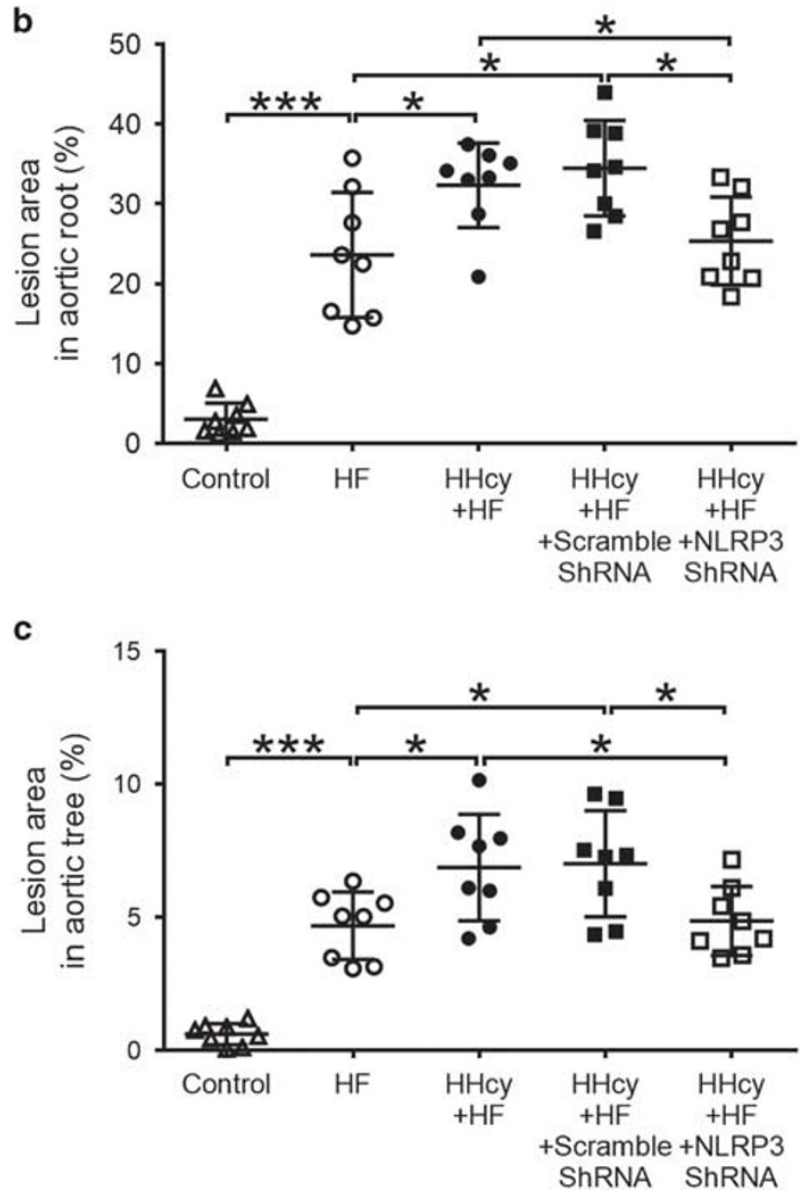

Figure 2 Lentiviral silencing of NLRP3 attenuated HHcy-accelerated atherosclerosis. ApoE ${ }^{-1-}$ mice were fed regular chow (control), high fat (HF) diet or HF plus high methionine (HF+HM) diet to induce HHcy for 10 weeks. The NLRP3 shRNA and scrambled shRNA were injected via tail vein twice at the second and the sixth week after HF+HM diet. Mouse aortas and hearts were harvested. Histologic analysis of aortic root plaque and en face analysis of aortic lesion are shown. (a) Oil red O staining showing the atherosclerotic lesions in aortic root (upper panel) and in the gross appearance of mouse aortic tree (lower panel). (b, c) Quantitative analysis of atherosclerotic lesion area in the aortic root and in the aortic tree. HHcy, hyperhomocysteinemia. $n=8 . * P<0.05, * * * P<0.001$.

Figure 3 Lentiviral silencing of NLRP3 inhibited HHcy-induced activation of NLRP3 inflammasomes and macrophages infiltration into atherosclerotic lesion. ApoE ${ }^{-1-}$ mice were fed regular chow (control), high fat (HF) diet or HF plus high methionine (HF+HM) diet to induce HHcy for 10 weeks. The NLRP3 shRNA and 31/33 scrambled shRNA were injected via tail vein twice at the second and the sixth week after HF+HM diet. Blockage efficiency was detected by protein expression of NLRP3 and activation of caspase-1 and IL-1 $\beta$. Serial sections of aortic roots were harvested and double

immunofluorescent stained. (a, b) Representative blots and quantitative analysis showing the expression of NLRP3 protein and the activation of caspase1 and IL-1 $\beta$ in aorta. $n=3 .{ }^{*} P<0.05,{ }^{*} P<0.01$. (c-e) Representative confocal microscopic images showing colocalization of NLRP3 (red) with ASC (green) (c), NLRP3 (red) with caspase-1 (green) (d) and NLRP3 (red) with MOMA-2 (green) (e) in atherosclerotic lesions of aortic root sections. cas-1, caspase-1; HHcy, hyperhomocysteinemia. 
Figures $3 \mathrm{c}$ and $\mathrm{d}$, in mice with the regular diet, no obvious expression of NLRP3, ASC and caspase-1 was detected within aortic root sections by confocal microscopy. In mice with the HF diet, NLRP3, ASC and caspase-1 were expressed at low levels, and little colocalization of these molecules could be observed. HHcy increased the co-expression of NLRP3, ASC and caspase-1 and colocalization of these molecules in aortic root plaque, as shown by yellow spots or patches in $\mathrm{HF}+\mathrm{HM}$ diet mice. Moreover, increased expression of NLRP3 and cleavage of caspase- 1 and IL- $1 \beta$

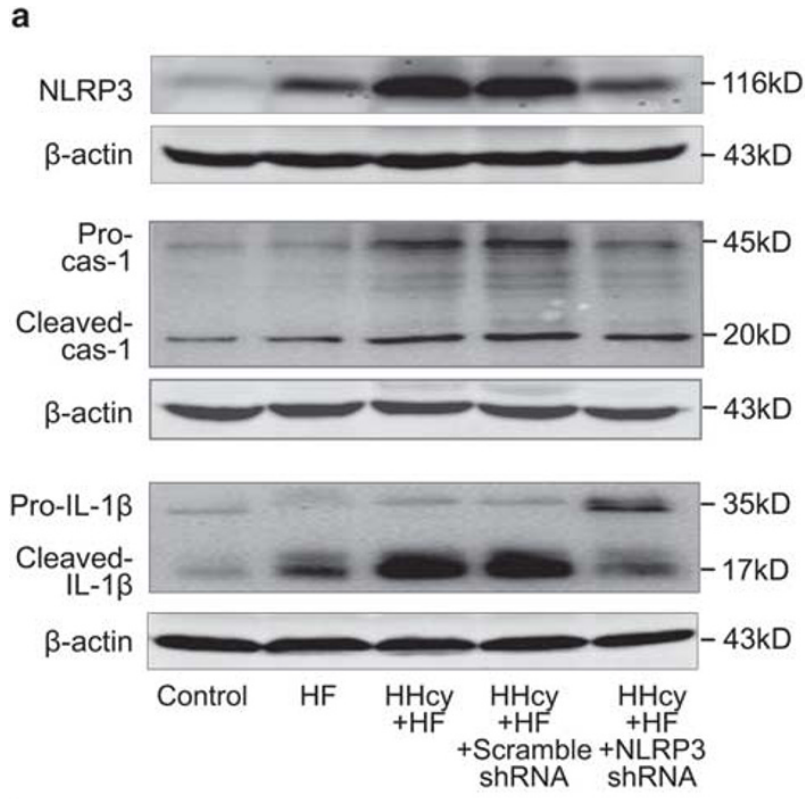

c

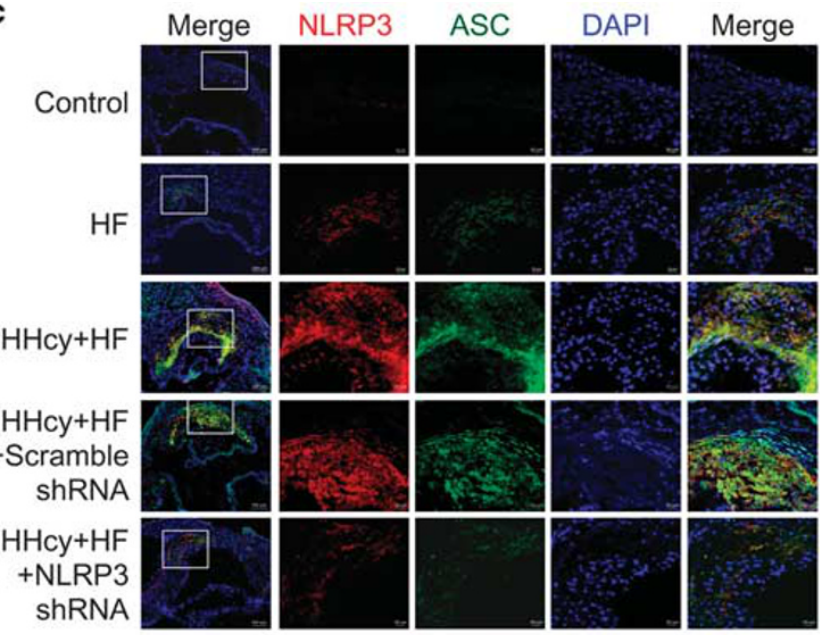

b
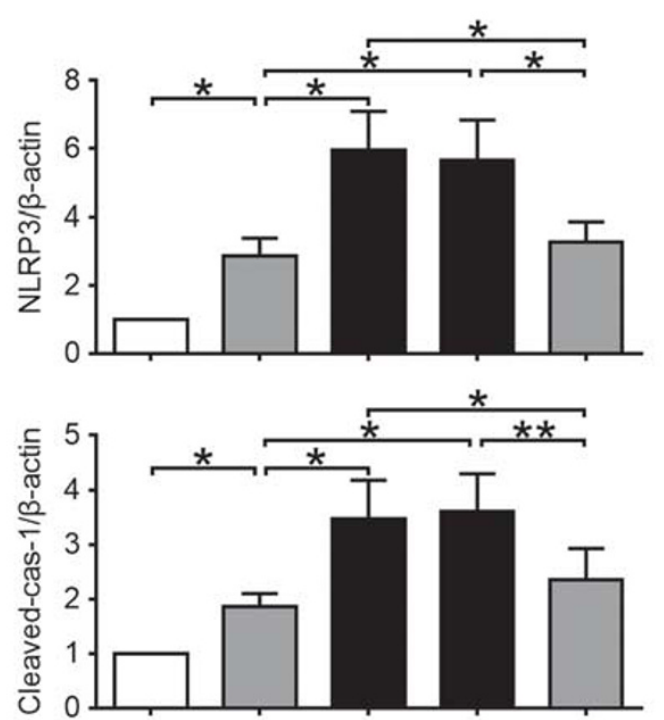

d

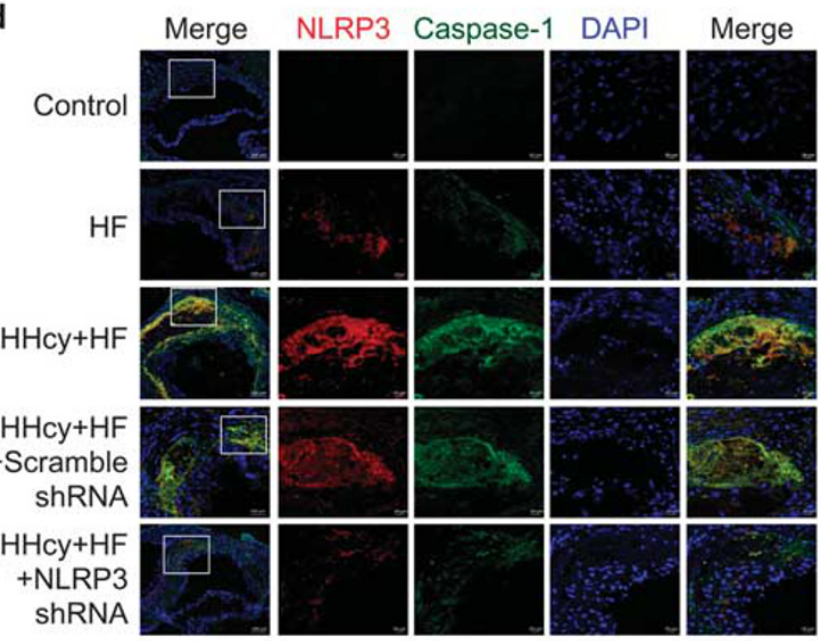

e
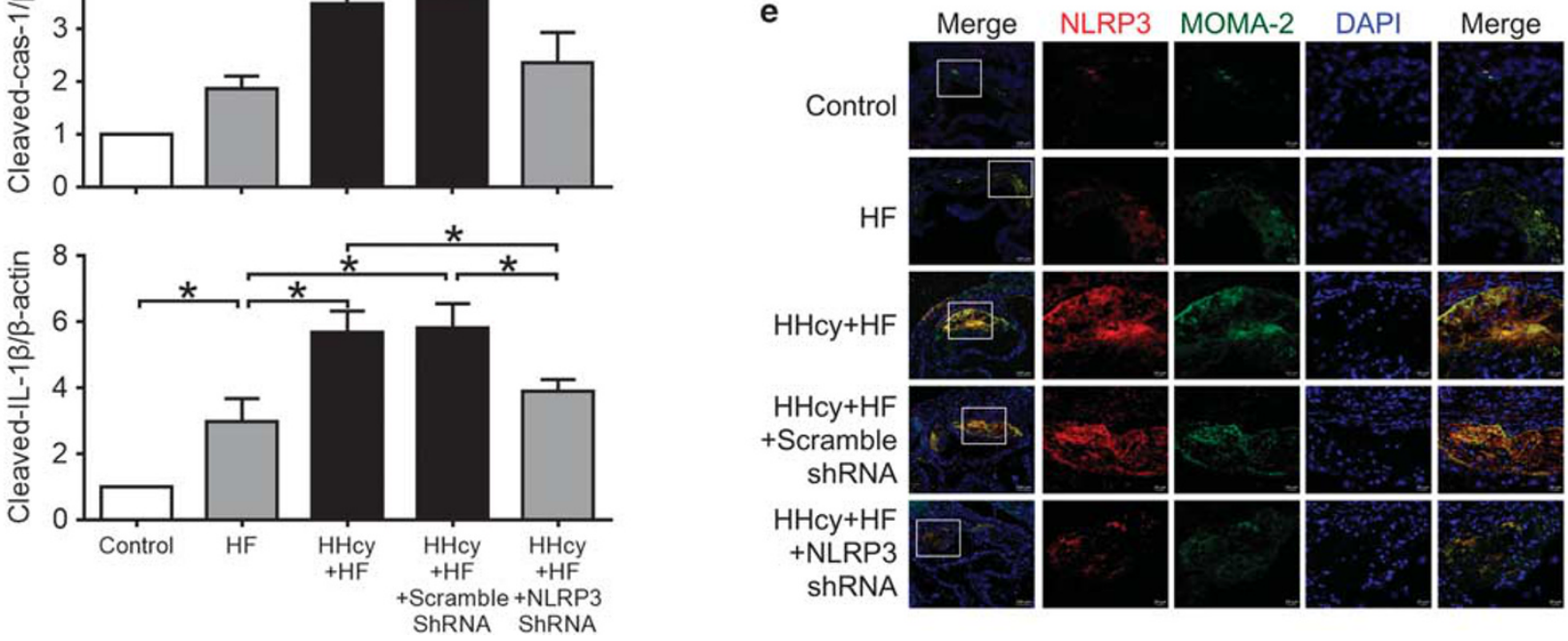

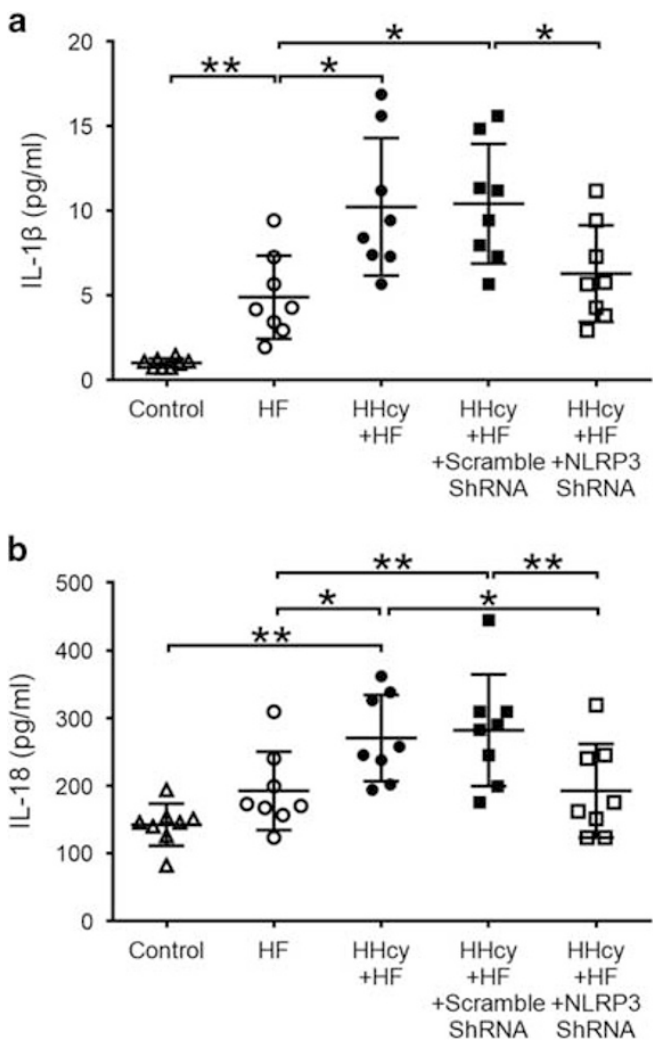

Figure 4 Lentiviral silencing of NLRP3 decreased plasma levels of IL-1 $\beta$ and $\mathrm{IL}-18$ induced by $\mathrm{HHcy}$. ApoE ${ }^{-/-}$mice were fed regular chow (control), high fat (HF) diet or HF plus high methionine (HF+HM) diet to induce HHcy for 10 weeks. The NLRP3 shRNA and scrambled shRNA were injected via tail vein twice at the second and the sixth week after $\mathrm{HF}+\mathrm{HM}$ diet. Plasma levels of IL-1 $\beta$ (a) and IL-18 (b) were measured by multiplexed immunoassay. HHcy, hyperhomocysteinemia. $n=8$. ${ }^{*} P<0.05$, ${ }^{*} P<0.01$

were observed in HHcy mice (Figures $3 \mathrm{a}$ and $\mathrm{b}$ ), indicating activation of NLRP3 inflammasome in the aorta. Importantly, NLRP3 shRNA substantially suppressed HHcy-induced colocalization of NLRP3 with ASC or with caspase-1, the expression of NLRP3, and cleavage of caspase-1 and IL- $1 \beta$ (Figures 3a-d), namely, the activation of NLRP3 inflammasomes induced by HHcy was blocked by NLRP3 gene silencing.

\section{Lentiviral Silencing of NLRP3 Inhibited Increased Plasma Levels of IL-1 $\beta$ and IL-18 Induced by HHcy}

Plasma levels of proinflammatory cytokines IL- $1 \beta$ and IL-18 were increased significantly in HHcy mice compared with mice fed regular diet or HF diet (Figures $4 \mathrm{a}$ and $\mathrm{b}$ ). Consistent with decreased co-expression of NLRP3 inflammasome molecules, HHcy-induced IL-1 $\beta$ and IL-18 were significantly reduced by NLRP3 shRNA (Figures $4 \mathrm{a}$ and b). These observations likely indicate an important role of NLRP3 inflammasomes in HHcy-induced inflammation.

\section{Activation of NLRP3 Inflammasomes Induced by HHcy Contributed to Macrophage Recruitment into Atherosclerotic Lesions}

To explore whether HHcy-induced NLRP3 inflammasome activation contributes to recruitment of macrophages in aorta, double immunofluorescent staining with anti-NLRP3 and MOMA-2 (monocyte and macrophage marker) antibodies were performed. As expected, macrophage infiltration was observed in the atherosclerotic lesions in mice with $\mathrm{HF}$ diet, and largely enhanced in HHcy mice. Enhanced NLRP3positive areas were clearly associated with the presence of macrophages in atherosclerotic lesions, whereas NLRP3 shRNA prevented the accumulation of macrophages in atherosclerotic lesions induced by HHcy (Figure 3e). These data suggest that HHcy-aggravated macrophage infiltration into atherosclerotic lesions, which was potentially suppressed by NLRP3 silencing with lentivirus. It is plausible that HHcyinduced activation of NLRP3 inflammasomes contributed to macrophage recruitment into atherosclerotic lesion.

\section{Hcy Activated NLRP3 Inflammasomes and Increased the Production of IL-1 $\beta$ and IL-18 in Macrophages}

THP-1-derived macrophages were incubated with different concentrations of Hcy for $24 \mathrm{~h}$. Western blot analysis showed that Hcy significantly increased the expression of NLRP3 protein in a dose-dependent manner in macrophages (Figure 5a). Double immunofluorescent staining showed that NLRP3, ASC and caspase-1 were expressed at a low level with little colocalization under normal conditions. Treatment with Hcy markedly increased the expression of NLRP3, ASC and caspase-1, and induced colocalization of these molecules (Figure 6a), suggesting an activation of NLRP3 inflammasomes by Hcy in cultured macrophages. Moreover, treatment with Hcy for $24 \mathrm{~h}$ significantly increased the levels of IL- $1 \beta$ and IL-18 in cultured supernatant in a dose-dependent manner (Figures $5 \mathrm{~b}$ and $\mathrm{c}$ ).

\section{Hcy-Induced Activation of NLRP3 Inflammasomes and Production of IL-1 $\beta$ and IL-18 Were Abolished by NLRP3 Silence or Caspase-1 Inhibition}

To further investigate the role of NLRP3 inflammasome activation in Hcy-induced production of IL- $1 \beta$ and IL-18 in vitro, macrophages transfected with NLRP3 siRNA or nontargeting scrambled siRNA were subsequently incubated with Hcy. As shown in Figure 6b, the expression of NLPR3 protein was significantly inhibited by NLRP3 siRNA. NLRP3 siRNA significantly reduced Hcy-induced activation of NLRP3 inflammasomes and production of IL- $1 \beta$ and IL-18 in macrophages (Figures $6 a$ and c). Similarly, blockade of caspase-1 activity by Z-WEHD-FMK also significantly abolished Hcy-induced production of IL-1 $\beta$ and IL-18 (Figure 6c). These data indicate that Hcy-induced production of proinflammatory cytokines IL- $1 \beta$ and IL-18 was dependent on the activation of NLRP3 inflammasomes in vitro. 
a
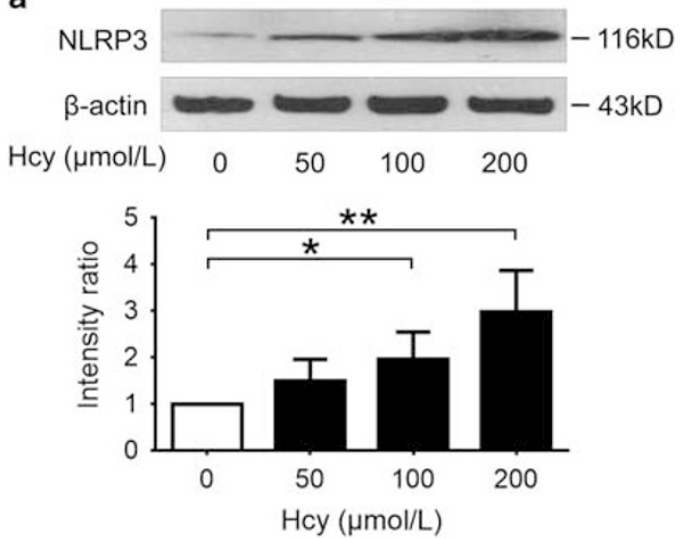

b

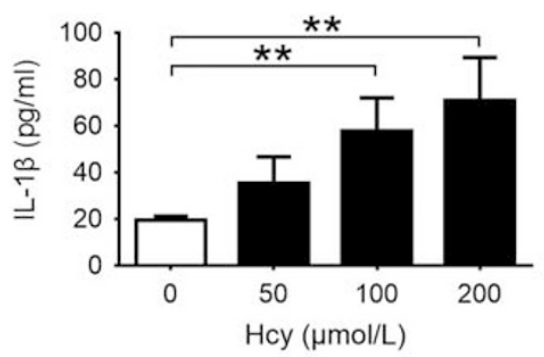

c

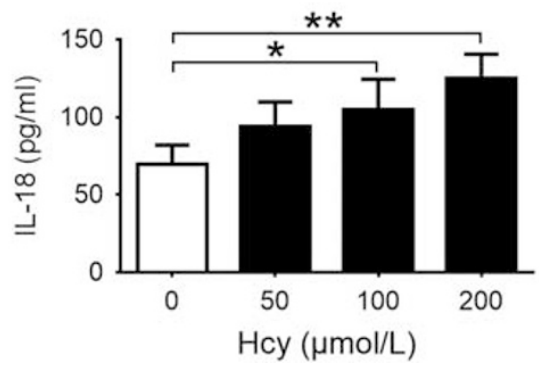

Figure 5 Hcy promoted the expression of NLRP3 protein and increased the production of IL-1 $\beta$ and IL-18 in macrophages. THP-1-differentiated macrophages were treated with indicates concentrations of Hcy for $24 \mathrm{~h}$. Cells were subsequently harvested for western blotting, and supernatants were $32 / 33$ collected for ELISA of IL-1 $\beta$ and IL-18. Data are representative of three independent experiments. (a) Representative blots and quantitative analysis of NLRP3 to $\beta$-actin. (b, c) Levels of IL-1 $\beta$ and IL-18 in cultured supernatant were measured by ELISA. Hcy, homocysteine. ${ }^{*} P<0.05,{ }^{* *} P<0.01$.

\section{Hcy Activated NLRP3 Inflammasomes via a ROS-Dependent Pathway}

We then investigated the role of ROS in Hcy-induced NLRP3 inflammasome activation. Our results show that ROS was increased markedly in macrophages after stimulation with Hcy, which was blocked by pretreatment with antioxidant NAC (Figure 7b). Interestingly, NAC also blocked the expression of NLRP3 protein, colocalization of NLRP3 inflammasome molecules, and production of IL- $1 \beta$ and IL-18 induced by Hcy in macrophages (Figures $7 \mathrm{a}, \mathrm{c}$ and d). These data indicate that Hcy activated NLRP3 inflammasomes in macrophages via ROS-dependent pathway.

\section{NAC Attenuated HHcy-Induced NLRP3 Inflammasome Activation and Atherosclerotic Lesion Formation in ApoE $^{-/-}$Mice}

To further examine the role of ROS in HHcy-induced activation of NLRP3 inflammasomes in vivo, HHcy mice were i.p. injected with ROS scavenger NAC. Treatment with NAC inhibited the expression and colocalization of NLRP3, ASC and caspase- 1 in the atherosclerotic lesions of HHcy mice (Figures $8 \mathrm{a}$ and $\mathrm{b}$ ), and suppressed the cleavage of IL- $1 \beta$ in the aorta of HHcy mice (Figure 8d), indicating that ROS may have a role in HHcy-induced activation of NLRP3 inflammasome in the aorta. Consistent with this, HHcy-enhanced macrophage recruitment in the atherosclerotic lesions was also suppressed by NAC (Figure 8c). Importantly, treatment with NAC attenuated HHcy-induced atherosclerosis in the aortic root (Figure 8e). These observations suggest that HHcy may induce activation of NLRP3 inflammasomes via a ROS-dependent pathway, which could at least partially contribute to HHcy-aggravated inflammation and atherosclerosis in $\mathrm{apoE}^{-1-}$ mice.

\section{DISCUSSION}

In this study, we examined the contributing role and molecular mechanisms of NLRP3 inflammasomes in HHcyaggravated atherosclerosis. To our knowledge, we have provided the first in vivo evidence that the activation of NLRP3 inflammasomes modulates the proinflammatory effect of HHcy, thereby contributing to atherogenesis. A ROS scavenger prevented Hcy-induced activation of NLRP3 inflammasome in vitro and in vivo, and suppressed atherosclerotic lesion formation in HHcy mice, indicating that ROS has an important role in HHcy-induced activation of NLRP3 inflammasome. This finding provides new insight into HHcyassociated cardiovascular diseases and indicates a new potential molecular target for the therapy of these diseases.

It was first reported that NLRP3 inflammasomes are activated by cholesterol crystals and are required for high cholesterol diet-induced atherosclerosis in 2010, ${ }^{12}$ highlighting the prominent role of NLRP3 inflammasomes in the development of atherosclerosis. As a critical risk of atherogenesis, oxidized low-density lipoprotein was also demonstrated to activate the NLRP3 inflammasomes. ${ }^{13} \mathrm{~A}$ recent study showed that NLRP3 expression elevated in the monocytes of patients with coronary artery disease. ${ }^{21}$ These findings indicate that NLRP3 inflammasomes have a crucial role in the development of atherosclerosis. HHcy is an independent risk factor for cardiovascular diseases. Recent evidence suggests that HHcy enhances vascular inflammation and accelerates atherosclerosis. ${ }^{3}$ However, the mechanisms underlying HHcy-induced inflammation remain largely unknown, and the effect of NLRP3 inflammasomes on $\mathrm{HHcy}$-induced atherosclerosis has not been investigated.

In our experiments, a HM diet was administered to establish HHcy model in apoE $\mathrm{E}^{-/-}$mice. In healthy individuals, plasma Hcy is between 5 and $10 \mu \mathrm{mol} / \mathrm{l}$. Patients with 
a
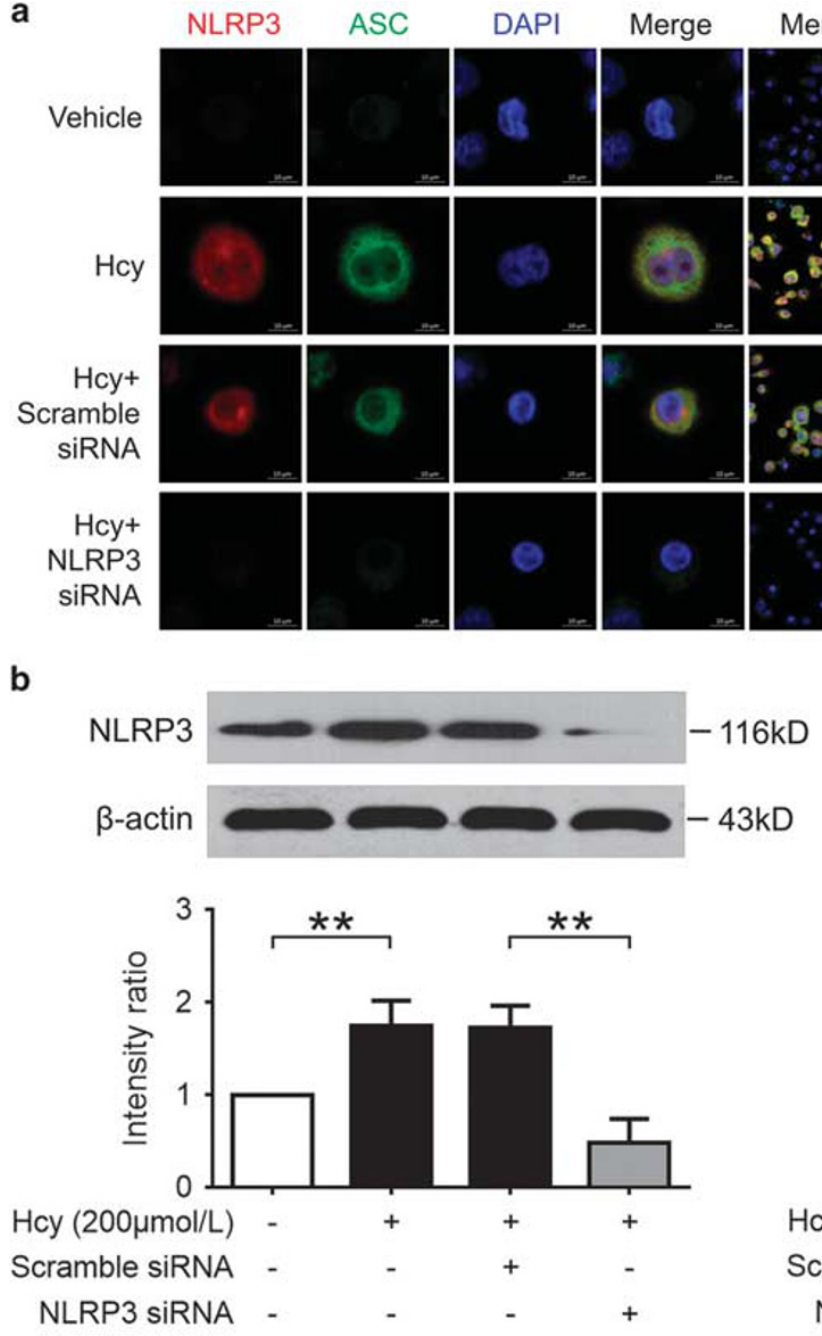

C
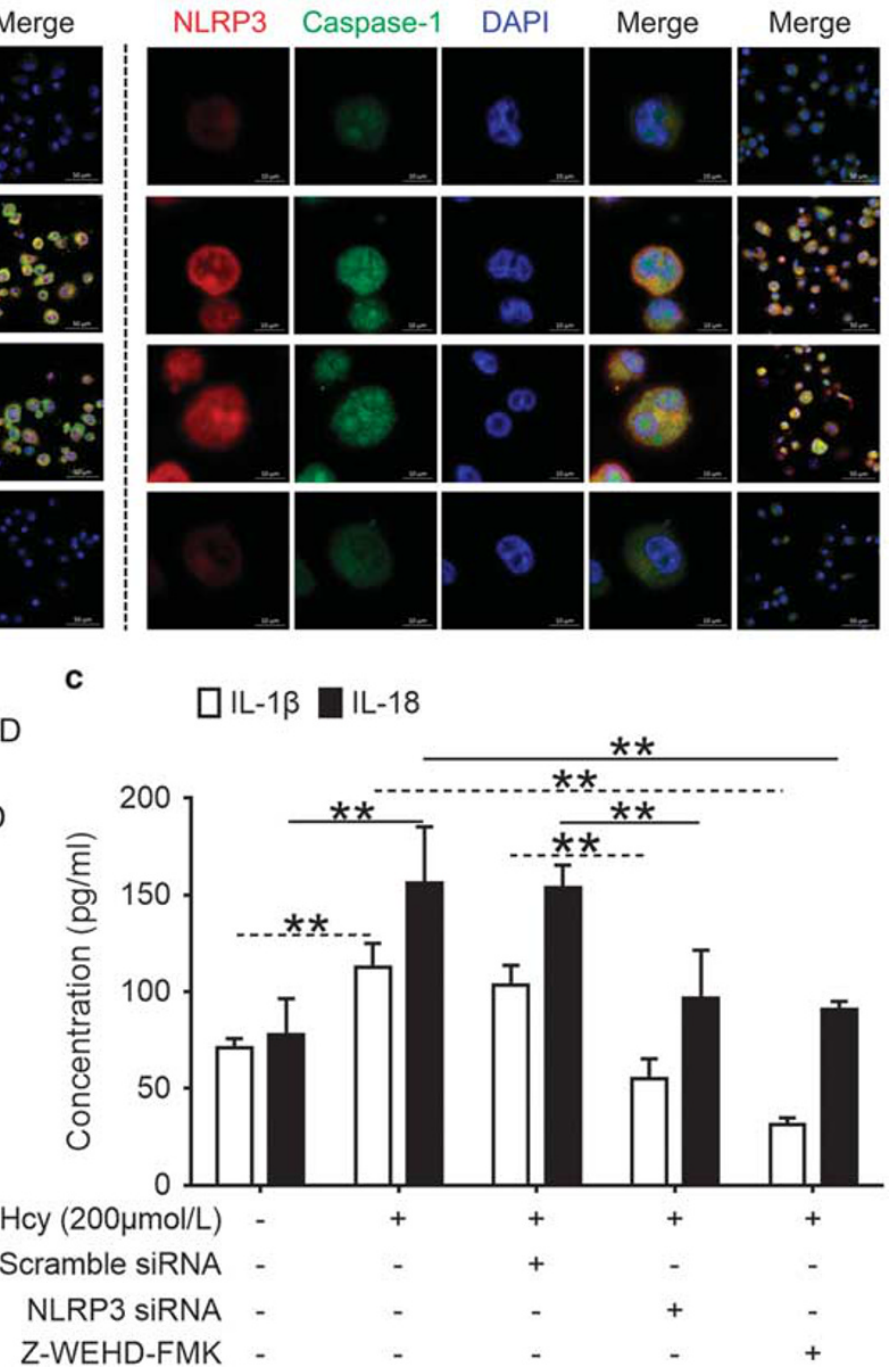

Figure 6 NLRP3 silence or caspase-1 inhibition abolished Hcy-induced activation of NLRP3 inflammasomes and production of IL-1 $\beta$ and IL-18. THP-1differentiated macrophages transfected with NLRP3 siRNA or scrambled siRNA were treated with Hcy $(200 \mu$ mol/l) for $24 \mathrm{~h}$, or treated with Hcy $(200 \mu \mathrm{mol} / \mathrm{l})$ with the presence or absence of caspase-1 inhibitor Z-WEHD-FMK $(20 \mu \mathrm{mol} / \mathrm{l})$ for $24 \mathrm{~h}$. Data are representative of three independent experiments. (a) Representative confocal microscopic images showing colocalization of NLRP3 (red) with ASC (green) or NLRP3 (red) with caspase-1 (green) in macrophages. (b) Representative blots and quantitative analysis of NLRP3 to $\beta$-actin. (c) Levels of IL-1 $\beta$ and IL-18 in cultured supernatant were measured by ELISA. Hcy, homocysteine. ${ }^{* *} P<0.01$.

plasma Hcy level $>10 \mu \mathrm{mol} / \mathrm{l}$ are at increased risk for vascular disorders. ${ }^{22,23}$ The $\mathrm{HF}+\mathrm{HM}$ diet induced moderate HHcy $(27.9 \pm 8.2 \mu \mathrm{mol} / \mathrm{l})$ in apoE $\mathrm{E}^{-1-}$ mice, comparable to the HHcy observed in cardiovascular patients, thus the observed plasma Hcy in our study was highly clinically relevant. Zhang et al reported the same formula HF+HM diet (TD08029, Harlan Teklad) induced severe HHcy $(244.6 \pm 50.4 \mu \mathrm{mol} / \mathrm{l})$ in $\mathrm{Ldlr}^{-/-} \mathrm{Cbs}^{-/+}$mice. ${ }^{3}$ As cystathionine $\beta$-synthase (CBS) is the key enzyme in the transsulfuration pathway that converts Hcy to cysteine, $\mathrm{Ldlr}^{-1-} \mathrm{Cbs}^{-/+}$mice developed severe HHcy, which resembled CBS-deficient humans. Hyperlipidemia was observed in mice fed HF diet and in HHcy mice induced by $\mathrm{HF}+\mathrm{HM}$ diet (the $\mathrm{HF}+\mathrm{HM}$ diet is the HF diet with up to $2 \%$ methionine), which is applicable to severe hyperlipidemia in humans. ${ }^{3}$
In this study, HF diet induced activation of NLRP3 inflammasomes, as seen in increased co-expression and colocalization of NLRP3 with ASC or caspase-1, as well as the cleavage of caspase- 1 and IL- $1 \beta$ in the aorta. This finding is consistent with a previous report showing that hyperlipidemia induced the expression and cleavage of caspase- 1 and IL- $1 \beta$ in the aorta of mice fed a HF diet. ${ }^{24}$ The HF diet induced atherosclerosis in apoE $\mathrm{E}^{-/-}$mice, and which was aggravated by moderate HHcy. In parallel with increased atherosclerotic lesions, we also found that the co-expression and colocalization of NLRP3 with ASC or caspase-1 in atherosclerotic lesions, expression of NLRP3 and cleaved caspase- 1 and IL- $1 \beta$ in the aorta, were markedly increased in HHcy mice compared with those in mice with HF diet. Increased levels of cleaved caspase- 1 and IL- $1 \beta$ may be 
a
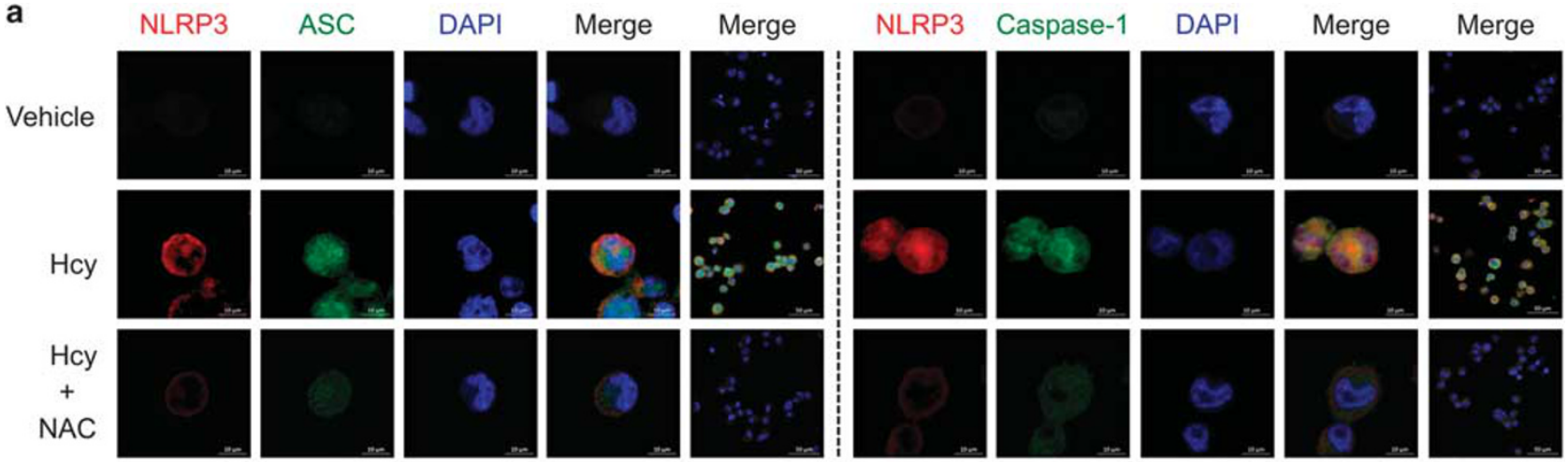

b

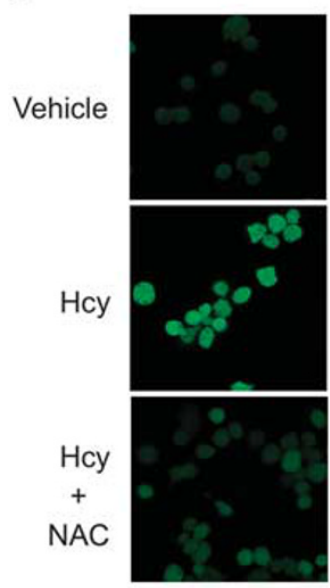

C
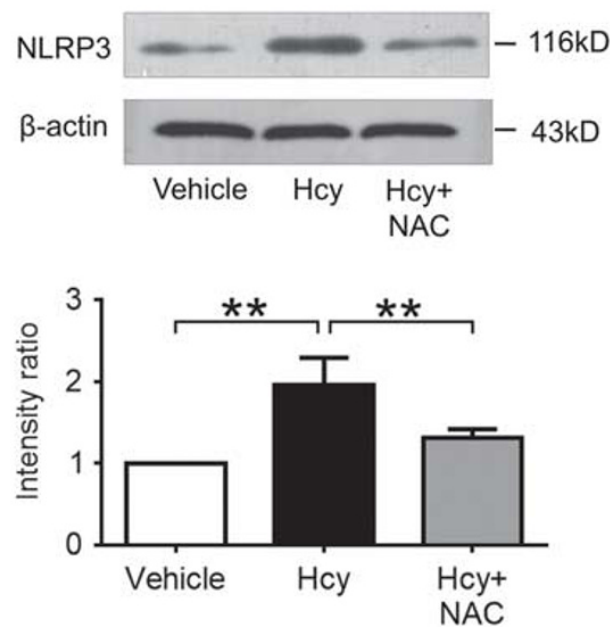
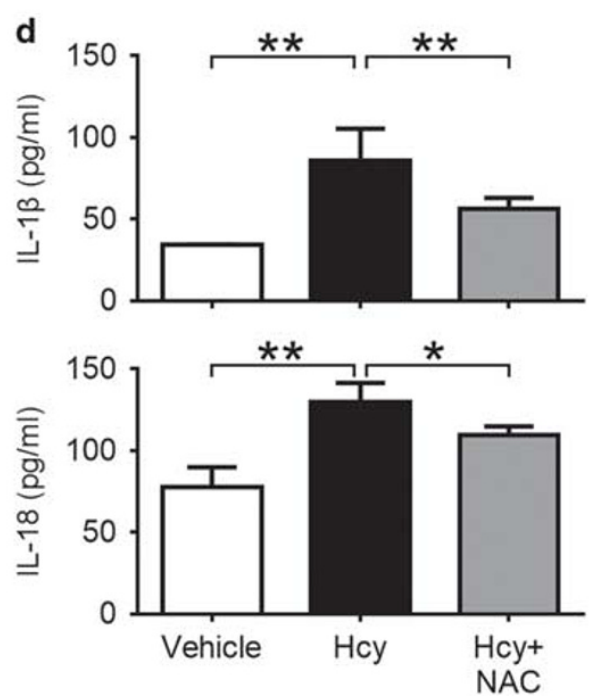

Figure 7 Hcy activated NLRP3 inflammasomes in a ROS-dependent pathway. THP-1-differentiated macrophages were preincubated with NAC (50 mmol/ l) for $1 \mathrm{~h}$, then treated with $200 \mu \mathrm{mol} / \mathrm{l} \mathrm{Hcy}$ for $0.5 \mathrm{~h}(\mathbf{b})$ or $24 \mathrm{~h}(\mathbf{a}, \mathbf{c}, \mathbf{d})$. Intracellular ROS levels were measured using a cell-permeable fluorescent probe, 2'7'-dichlorofluorescein diacetate (DCFH-DA). Data are representative of three independent experiments. (a) Representative confocal microscopic images showing colocalization of NLRP3 (red) with ASC (green), or NLRP3 (red) with caspase-1 (green) in macrophages. (b) Representative confocal microscopic images showing ROS accumulation in macrophages. (c) Representative blots and quantitative analysis of NLRP3 to $\beta$-actin. (d) Levels of IL-1 $\beta$ and IL-18 in cultured supernatant were measured by ELISA. Hcy, homocysteine; NAC, N-acetyl-L-cysteine. ${ }^{*} P<0.05,{ }^{* *} P<0.01$.

attributed to either increased activation of inflammasomes or increased expression of precursors (pro-caspase-1 and pro-IL-1 $\beta$ ). Our results indicate that both of these conditions contributed to the increased levels of cleaved caspase-1 and IL-1 $\beta$ (Figures $3 \mathrm{a}$ and $\mathrm{b}$ ), as the activation of NLRP3 inflammasome produces active caspase-1, which transcriptionally activates NF- $\mathrm{kB}$-dependent gene expression, for example, proinflammatory cytokines pro-IL- $1 \beta$ and TNF- $\alpha$. In addition, plasma levels of IL-1 $\beta$ and IL-18 were substantially increased in HHcy mice, accompanied with increased expression of caspase- 1 and IL- $1 \beta$ in the aorta. These results strongly support the conception that HHcy induces NLRP3 inflammasome activation.

To further examine the role of NLRP3 inflammasome activation in HHcy-induced inflammation and atherosclerosis, NLRP3 shRNA or a scrambled shRNA viral suspension was injected to HHcy mice. Our results showed that NLRP3
shRNA substantially suppressed HHcy-induced colocalization of NLRP3 with ASC or with caspase-1, the expression of NLRP3, and cleavage of caspase-1 and IL-1 $\beta$. Although little evidence shows that ASC or caspase-1 protein expression is regulated directly by NLRP3, it has been reported that the expression of ASC is increased in the neointima after vascular injury in mice and it is colocalized with macrophages deriving from the bone marrow. ${ }^{25}$ Consistent with this finding, we found that HHcy promoted macrophage recruitment into atherosclerotic lesion, which was reduced when NLRP3 is inhibited by shRNA. The observed downregulation of ASC may be associated with reduced macrophage recruitment in the aortas of mice with NLRP3 shRNA. Furthermore, Miggin et al $^{26}$ have demonstrated a role for caspase-1 in inflammation beyond its ability to process pro-IL-1 $\beta$ or pro-IL-18, implicating it in the regulation of many proinflammatory genes via NF- $\mathrm{BB}$ activation. This feedback will strength 


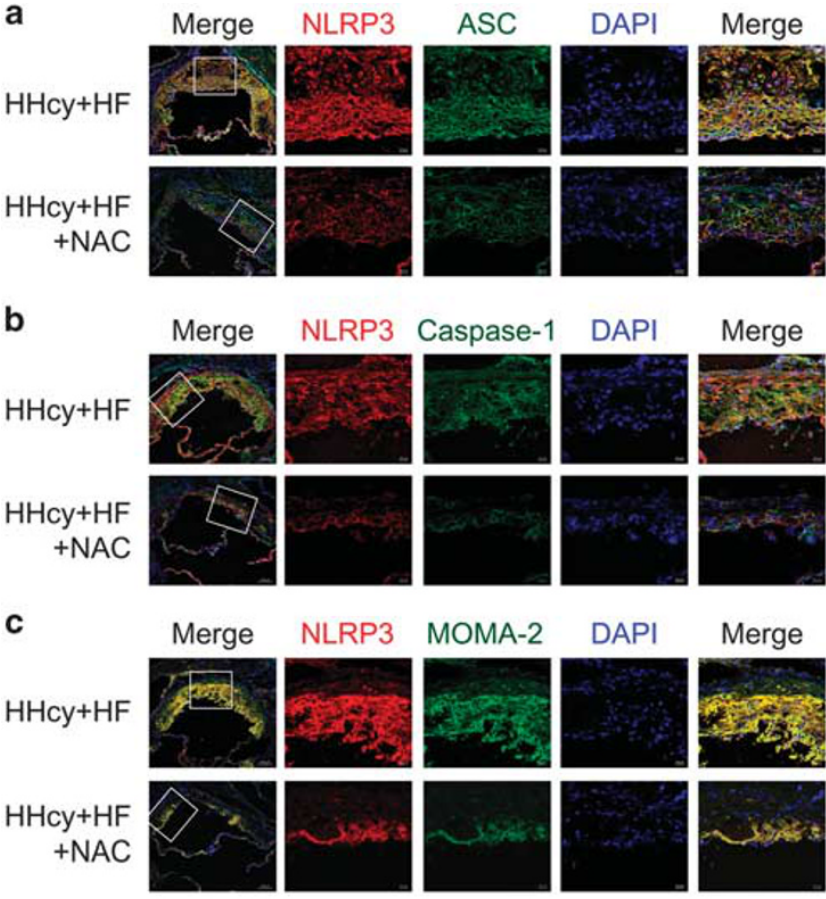

d

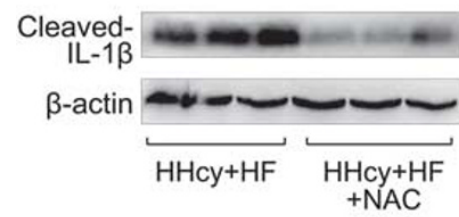

e

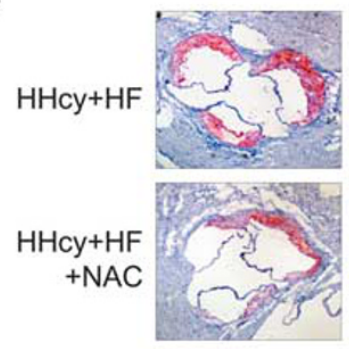

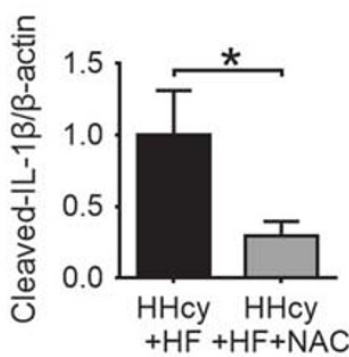

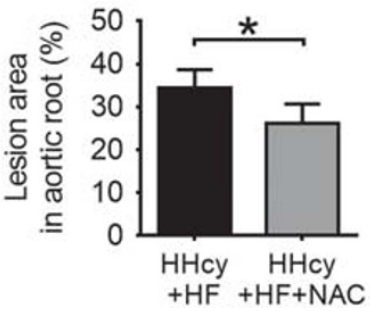

Figure 8 NAC attenuated HHcy-induced NLRP3 inflammasome activation and atherosclerotic lesion formation in apoE $\mathrm{E}^{-/-}$mice. $\mathrm{ApoE}^{-/-}$mice were fed high-fat plus high methionine $(\mathrm{HF}+\mathrm{HM})$ diet to induce $\mathrm{HHcy}$ for 10 weeks. Mice were given a daily i.p. injection of either saline $(n=6)$ or antioxidant NAC (Sigma, $20 \mathrm{mg} / \mathrm{kg} / \mathrm{day}, n=8$ ) three times per week on alternate days over 10 weeks of $\mathrm{HF}+\mathrm{HM}$ diet. Mouse aortas and hearts were harvested. Serial sections of aortic roots were harvested and double immunofluorescent stained or stained with Oil red O. Representative confocal microscopic images showing colocalization of NLRP3 (red) with ASC (green) (a), NLRP3 (red) with caspase-1 (green) (b) and NLRP3 (red) with MOMA-2 (green) (c) in atherosclerotic lesion of aortic root sections. (d) Representative blots and quantitative analysis showing the cleaved IL- $1 \beta$ in aorta. (e) Oil red $O$ staining showing the atherosclerotic lesions and quantitative analysis of atherosclerotic lesion area in the aortic root. HHcy, hyperhomocysteinemia; NAC, $N$-acetyl-L-cysteine. ${ }^{*} P<0.05$.

priming signals functioning upstream of ASC, ${ }^{27}$ and may eventually affect the expression of inflammasome components including ASC.

Consistent with the inhibition of NLRP3 inflammasomes, HHcy-aggravated atherosclerotic lesion in the whole aorta and aortic root were significantly attenuated by lentiviral silencing of NLRP3. Correspondingly, plasma proinflammatory cytokines IL- $1 \beta$ and IL-18 were markedly decreased by lentiviral silencing of NLRP3. Taken together, these data suggest that NLRP3 inflammasomes had a critical role in HHcy-induced inflammation and atherosclerosis. It has been reported that NLRP3 inflammasomes formation and activation are important molecular mechanisms triggering podocyte injury and resulting in glomerulosclerosis in HHcy mice induced by a folate-free diet. $^{20}$ Wang et al recently demonstrated that HHcy activated NLRP3 inflammasomes in an abdominal aortic aneurysm mouse model on top of regular diet, which indicates that Hcy alone induced activation of the NLRP3 inflammasome in vivo. ${ }^{28}$ As it is hard to induce prominent spontaneous atherosclerosis in mice even with apoE gene deficiency, we therefore set up an $\mathrm{HH}$ cy mice model with $\mathrm{HF}+\mathrm{HM}$ diet to examine whether HHcy can activate NLRP3 inflammasome and promote atherogenesis. It was widely accepted to investigate HHcy-related atherogenesis and mechanisms on top of HF diet. $^{3,29}$ This study is in agreement with these findings and provides further evidence demonstrating that the activation of NLRP3 inflammasomes contributes to HHcy-accelerated atherosclerosis.

Macrophage infiltration is one major process involved in the development of atherosclerosis. ${ }^{30}$ Inflammasome components and proinflammatory caspases are constitutively expressed in macrophages, ${ }^{31}$ which indicates an important role of macrophages in inflammasome-mediated changes. Here we found that MOMA-2-positive area was increased in the atherosclerotic lesions of HHcy mice, suggesting macrophage recruitment was enhanced in the atherosclerotic lesions by HHcy. Confocal microscopic analysis also demonstrated that NLRP3-positive areas were equally associated with the presence of adjacent macrophages in atherosclerotic lesions of HHcy mice. In addition, NLRP3 silence substantially suppressed HHcy-induced macrophage infiltration, indicating NLRP3 inflammasomes mediated macrophage recruitment induced by HHcy in aortic wall.

To detect whether Hcy regulates NLRP3 inflammasomes in vitro, we further explored the effect of Hcy on NLRP3 inflammasome activation in cultured macrophages. We observed that Hcy induced the expression of NLRP3 protein and increased the production of IL-1 $\beta$ and IL-18 in macrophages. In addition, we found that Hcy increased the colocalization of NLRP3 with ASC or caspase-1 in macrophages, indicating the aggregation or assembly of these inflammasome molecules, namely, the activation of NLRP3 inflammasomes in macrophages. Importantly, Hcy-induced activation of NLRP3 inflammasomes and production of IL- $1 \beta$ 
and IL-18 were substantially blocked by NLRP3 gene silencing. Consistent with these findings, production of IL- $1 \beta$ and IL-18 induced by Hcy was also blocked by Z-YVAD-FMK, a specific caspase-1 inhibitor. Therefore, it is reasonable to speculate that NLRP3 inflammasomes mediated Hcy-induced proinflammatory effect.

Three models for activation of the NLRP3 inflammasomes have been proposed, these are lysosome rupture model, ion channel model and ROS model. ${ }^{16}$ Accumulating evidence support the central role of ROS in NLRP3 inflammasome activation. ${ }^{16,32}$ Many endogenous and exogenous inflammasomes activators can promote ROS generation. Activation of the NLRP3 inflammasomes by increased ROS was the most widely accepted and considered to be the most plausible mechanism. Hcy can be autooxidized with another Hcy molecule, and generate the disulfide and ROS. It is therefore reasonable to speculate that Hcy can activate NLRP3 inflammasomes by ROS model.

Oxidation has been proposed as a primary biochemical mechanism responsible for Hcy pathogenesis. Studies showed that high level of Hcy is able to increase the ROS production in vasculature cells. ${ }^{33}$ Therefore, we hypothesized that ROS-dependent pathway may be involved in Hcy-induced activation of NLRP3 inflammasomes. In our experiments, we observed that Hcy enhanced the accumulation of ROS in macrophages. Blockage of ROS with NAC reversed the activation of NLRP3 inflammasomes and blocked the production of IL- $1 \beta$ and IL-18 induced by Hcy. To further examine the role of ROS in HHcy-induced activation of NLRP3 inflammasomes and atherosclerosis in vivo, HHcy mice were treated with antioxidant NAC. In consistent with the in vitro data, NAC markedly suppressed HHcy-induced expression and colocalization of NLRP3, ASC and caspase-1 in the atherosclerotic lesion, and suppressed the cleavage of IL- $1 \beta$ in the aorta. In parallel with the inhibition of NLRP3 inflammasomes, HHcy-enhanced macrophage recruitment and atherogenesis were also significantly attenuated by NAC treatment, which was consistent with a previous study. ${ }^{17} \mathrm{NAC}$ treatment did not significantly modify the plasma Hcy and lipid profiles (data not shown). It is therefore possible that HHcy-induced activation of NLRP3 inflammasomes via ROS-dependent pathway, which at least partially contributed to HHcy-aggravated inflammation and atherosclerosis in $\operatorname{apoE}^{-/-}$mice.

A large number of studies suggest a model in which one of the crucial elements for NLRP3 activation is the generation of ROS. It is believed that ROS has an important role in the assembly and activation of NLRP3 inflammasomes (ie, signal 2). ${ }^{16}$ In this study, we found that ROS scavenger NAC not only attenuated Hcy-induced NLRP3 inflammasomes assembly, but also decreased the expression of its component molecules (ie, signal 1). Our data suggest that ROS modulate both steps (ie, priming and assembly) in the process of NLRP3 inflammasome activation. This result is consistent with a recent study that showed that NAC inhibited the expression of NLRP3 and ASC, and the cleavage of caspase-1 induced by lipopolysaccharide in lung. ${ }^{34}$

Several sources of ROS have been proposed to involve in the regulation of inflammation. These include nicotinamide adenine dinucleotide phosphate (NADPH) oxidase-derived ROS, ${ }^{35}$ mitochondrial-derived ROS, ${ }^{36}$ uncoupled nitric oxide synthase (NOS)-derived $\operatorname{ROS}^{37}$ and xanthine oxidase-derived ROS. ${ }^{38}$ Abais et $a l^{35}$ found that NADPH oxidase mediated the triggering of inflammasome activation in mouse podocytes and glomeruli during HHcy. Mitochondrial ROS was recently demonstrated to participate in Hcy-induced activation of inflammasomes in mouse macrophages. ${ }^{28}$ It was also reported that Hcy induces oxidative stress by uncoupling of NOS activity through reduction of tetrahydrobiopterin. ${ }^{39}$ Taken together, NADPH oxidase-derived ROS, mitochondrialderived ROS and uncoupled NOS-derived ROS may be involved in Hcy-associated NLRP3 inflammasome activation. Apparently further studies are warranted for a more thorough understanding of potential association between ROS and NLRP3 inflammasome activation.

In summary, we have demonstrated for the first time that the activation of NLRP3 inflammasomes has an essential role in HHcy-aggravated atherosclerosis. Our study provides a new understanding of the mechanisms driving HHcy-induced atherosclerosis. Targeting the activation of NLRP3 inflammasomes may be a promising therapeutic intervention to prevent HHcy-associated cardiovascular diseases.

\section{ACKNOWLEDGMENTS}

This work was supported by the National Natural Science Foundation of China (grant numbers 81570394 and 81370371), Guangdong Natural Science Foundation (grant number 2014A030313066) and Science and Technology Program of Guangdong (grant numbers 2015B090903063 and 2012B031800254). The authors thank Dr Weidong Wang for constructive comments on paper preparation.

\section{DISCLOSURE/CONFLICT OF INTEREST}

The authors declare no conflict of interest.

1. Hansson GK. Inflammation, atherosclerosis, and coronary artery disease. N Engl J Med 2005;352:1685-1695.

2. Zeng $X$, Dai J, Remick DG, Wang X. Homocysteine mediated expression and secretion of monocyte chemoattractant protein-1 and interleukin8 in human monocytes. Circ Res 2003;93:311-320.

3. Zhang D, Fang $P$, Jiang $X$, et al. Severe hyperhomocysteinemia promotes bone marrow-derived and resident inflammatory monocyte differentiation and atherosclerosis in LDLr/CBS-deficient mice. Circ Res 2012;111:37-49.

4. Martinon F, Burns K, Tschopp J. The inflammasome: a molecular platform triggering activation of inflammatory caspases and processing of prolL-beta. Mol Cell 2002;10:417-426.

5. Mariathasan S, Newton K, Monack DM, et al. Differential activation of the inflammasome by caspase- 1 adaptors ASC and Ipaf. Nature 2004:430:213-218.

6. Schroder K, Tschopp J. The inflammasomes. Cell 2010;140:821-832.

7. Davis BK, Wen H, Ting JP. The inflammasome NLRs in immunity, inflammation, and associated diseases. Annu Rev Immunol 2011;29: 707-735.

8. Masters SL, Dunne A, Subramanian SL, et al. Activation of the NLRP3 inflammasome by islet amyloid polypeptide provides a mechanism for enhanced IL-1 beta in type 2 diabetes. Nat Immunol 2010;11:897-904. 
9. Halle A, Hornung V, Petzold GC, et al. The NALP3 inflammasome is involved in the innate immune response to amyloid-beta. Nat Immunol 2008;9:857-865.

10. Cassel SL, Eisenbarth SC, lyer SS, et al. The Nalp3 inflammasome is essential for the development of silicosis. Proc Natl Acad Sci USA 2008;105:9035-9040.

11. Gasse P, Mary C, Guenon I, et al. IL-1R1/MyD88 signaling and the inflammasome are essential in pulmonary inflammation and fibrosis in mice. J Clin Invest 2007;117:3786-3799.

12. Duewell $\mathrm{P}$, Kono $\mathrm{H}$, Rayner $\mathrm{KJ}$, et al. NLRP3 inflammasomes are required for atherogenesis and activated by cholesterol crystals. Nature 2010;464:1357-1361.

13. Jiang $\mathrm{Y}$, Wang $\mathrm{M}$, Huang $\mathrm{K}$, et al. Oxidized low-density lipoprotein induces secretion of interleukin-1beta by macrophages via reactive oxygen species-dependent NLRP3 inflammasome activation. Biochem Biophys Res Commun 2012;425:121-126.

14. Zhou R, Tardivel A, Thorens B, et al. Thioredoxin-interacting protein links oxidative stress to inflammasome activation. Nat Immunol 2010;11:136-140.

15. Zheng F, Xing S, Gong Z, et al. Silence of NLRP3 suppresses atherosclerosis and stabilizes plaques in apolipoprotein E-deficient mice. Mediators Inflamm 2014;2014:507208.

16. Tschopp J, Schroder K. NLRP3 inflammasome activation: the convergence of multiple signalling pathways on ROS production? Nat Rev Immunol 2010;10:210-215.

17. Shimada $\mathrm{K}$, Murayama $\mathrm{T}$, Yokode $\mathrm{M}$, et al. $\mathrm{N}$-acetylcysteine reduces the severity of atherosclerosis in apolipoprotein E-deficient mice by reducing superoxide production. Circ J 2009;73:1337-1341.

18. Cao L, Lou X, Zou Z, et al. Folic acid attenuates hyperhomocysteinemiainduced glomerular damage in rats. Microvasc Res 2013;89:146-152.

19. Zhang Q, Li Q, Chen Y, et al. Homocysteine-impaired angiogenesis is associated with VEGF/VEGFR inhibition. Front Biosci, (Elite ed) 2012;4: 2525-2535.

20. Zhang $\mathrm{C}$, Boini KM, Xia M, et al. Activation of Nod-like receptor protein 3 inflammasomes turns on podocyte injury and glomerular sclerosis in hyperhomocysteinemia. Hypertension 2012;60:154-162.

21. Wang L, Qu P, Zhao J, et al. NLRP3 and downstream cytokine expression elevated in the monocytes of patients with coronary artery disease. Arch Med Sci 2014;10:791-800.

22. McCully KS. Homocysteine and vascular disease. Nat Med 1996;2: 386-389.

23. Stampfer MJ, Malinow MR, Willett WC, et al. A prospective study of plasma homocyst(e)ine and risk of myocardial infarction in US physicians. JAMA 1992;268:877-881.

24. Yin $Y$, Li $X$, Sha $X$, et al. Early hyperlipidemia promotes endothelial activation via a caspase-1-sirtuin 1 pathway. Arterioscler Thromb Vasc Biol 2015;35:804-816.

25. Yajima $\mathrm{N}$, Takahashi $\mathrm{M}$, Morimoto $\mathrm{H}$, et al. Critical role of bone marrow apoptosis-associated speck-like protein, an inflammasome adaptor molecule, in neointimal formation after vascular injury in mice. Circulation 2008;117:3079-3087.

26. Miggin SM, Palsson-McDermott E, Dunne A, et al. NF-kappaB activation by the Toll-IL-1 receptor domain protein MyD88 adapter-like is regulated by caspase-1. Proc Natl Acad Sci USA 2007;104:3372-3377.

27. Bauernfeind FG, Horvath G, Stutz A, et al. Cutting edge: NF-kappaB activating pattern recognition and cytokine receptors license NLRP3 inflammasome activation by regulating NLRP3 expression. J Immunol 2009;183:787-791.

28. Sun W, Pang Y, Liu Z, et al. Macrophage inflammasome mediates hyperhomocysteinemia-aggravated abdominal aortic aneurysm. J Mol Cell Cardiol 2015;81:96-106.

29. Zhang $D$, Jiang $X$, Fang $P$, et al. Hyperhomocysteinemia promotes inflammatory monocyte generation and accelerates atherosclerosis in transgenic cystathionine beta-synthase-deficient mice. Circulation 2009;120:1893-1902.

30. Moore KJ, Sheedy FJ, Fisher EA. Macrophages in atherosclerosis: a dynamic balance. Nat Rev Immunol 2013;13:709-721.

31. Yin $Y$, Yan $Y$, Jiang $X$, et al. Inflammasomes are differentially expressed in cardiovascular and other tissues. Int J Immunopathol Pharmacol 2009;22:311-322.

32. Zhou R, Yazdi AS, Menu $P$, et al. A role for mitochondria in NLRP3 inflammasome activation. Nature 2011;469:221-225.

33. Tsen CM, Hsieh CC, Yen CH, Lau YT. Homocysteine altered ROS generation and NO accumulation in endothelial cells. Chin J Physiol 2003:46:129-136

34. Yin N, Peng Z, Li B, et al. Isoflurane attenuates lipopolysaccharideinduced acute lung injury by inhibiting ROS-mediated NLRP3 inflammasome activation. Am J Transl Res 2016;8:2033-2046.

35. Abais JM, Zhang C, Xia M, et al. NADPH oxidase-mediated triggering of inflammasome activation in mouse podocytes and glomeruli during hyperhomocysteinemia. Antioxid Redox Signal 2013;18:1537-1548.

36. Misawa T, Takahama M, Kozaki $\mathrm{T}$, et al. Microtubule-driven spatial arrangement of mitochondria promotes activation of the NLRP3 inflammasome. Nat Immunol 2013;14:454-460.

37. Mishra BB, Rathinam VA, Martens GW, et al. Nitric oxide controls the immunopathology of tuberculosis by inhibiting NLRP3 inflammasomedependent processing of IL-1beta. Nat Immunol 2013;14:52-60.

38. Ives A, Nomura J, Martinon $\mathrm{F}$, et al. Xanthine oxidoreductase regulates macrophage IL1beta secretion upon NLRP3 inflammasome activation. Nat Commun 2015;6:6555.

39. He L, Zeng H, Li $F$, et al. Homocysteine impairs coronary artery endothelial function by inhibiting tetrahydrobiopterin in patients with hyperhomocysteinemia. Am J Physiol Endocrinol Metab 2010;299: E1061-E1065

This work is licensed under a Creative Commons Attribution-NonCommercial-ShareAlike $\quad \mathbf{4 . 0}$ International License. The images or other third party material in this article are included in the article's Creative Commons license, unless indicated otherwise in the credit line; if the material is not included under the Creative Commons license, users will need to obtain permission from the license holder to reproduce the material. To view a copy of this license, visit http://creativecommons.org/licenses/by-nc$\mathrm{sa} / 4.0 /$

(C) The Author(s) 2017 